CASO CLÍNICO

\title{
Complicaciones intracraneales de la otomastoiditis, presentación de caso clínico
}

\section{Intracranial complications of otomastoiditis, a case review}

Mario R. Prada-Bonilla1*, Flor de Lis Guzmán² y Nidia Escobar-Hernandez ${ }^{1}$

${ }^{1}$ Departamento de Radiología e Imagen; ${ }^{S}$ Servicio de Imagenología Diagnóstica y Terapéutica. Hospital General Dr. Manuel Gea González, Ciudad de México, México

\section{RESUMEN}

Las complicaciones intracraneales de la otomastoiditis siguen siendo un reto diagnóstico clínico y radiológico. Se presenta el caso de un paciente de sexo masculino, de 20 años de edad, quien se presentó con un cuadro clínico de traumatismo craneal y antecedente de síndrome de Cornelia de Lange. En estudios posteriores de tomografía computarizada y de resonancia magnética cerebral, se detectaron numerosas complicaciones infecciosas. En el presente artículo se revisan las principales características clínicas y las imágenes diagnósticas de las mismas, debido a que el retardo de su apropiado diagnóstico y la adecuada terapia, pueden llevar a incremento en la morbimortalidad.

Palabras clave: Otomastoiditis. Complicaciones intracraneales. Absceso cerebral. Tomografía computarizada. Resonancia magnética. 


\section{ABSTRACT}

Intracranial complications of otomastoiditis remain a clinical and radiological diagnostic challenge. We present the case of a male patient of 20 years of age, who presented with a clinical picture of head trauma and a history of Cornelia de Lange syndrome. In a subsequent brain computed tomography and magnetic resonance, numerous infectious complications were detected in the patient. In the present article, the main clinical characteristics and their diagnostic images are reviewed, since the delay in their appropriate diagnosis and adequate therapy can lead to an increase in morbidity and mortality.

Key words: Otomastoiditis. Intracranial complications. Brain abscess. Computed tomography. Magnetic resonance.

\section{INTRODUCCIÓN}

La mastoiditis coalescente es una complicación conocida de la otitis media aguda (OMA), que ha disminuido de forma muy importante a una incidencia tan baja como el $0.004 \%$ en la década de $1980^{1}$. Sin embargo, la OMA, asociada con complicaciones intratemporales, como otomastoiditis coalescente, absceso subperióstico, laberintitis, parálisis facial y petrositis, o asociada a complicaciones intracraneales, como meningitis, trombosis de los senos venosos durales, absceso epidural, empiema subdural y absceso encefálico, están en constante incremento debido a la resistencia antibiótica a las bacterias $^{2}$.

Se entiende como OMA la inflamación de la caja del oído medio, así como la trompa de Eustaquio y, en ocasiones, se asocia con un proceso inflamatorio de la mastoides, independiente de la causa que lo ocasione. Cuando este proceso da lugar a cambios en el mucoperiostio, nos encontramos ante una secuela que propiciaría el desarrollo de una complicación si el proceso infeccioso se extendiera a través del mismo ${ }^{3}$. La incidencia de infecciones óticas agudas y de mastoiditis varía en función de la edad del paciente. Los principales gérmenes aislados en las infecciones agudas son el Haemophilus influenzae, Streptococcus pneumoniae y Moraxella catarrhalis. Durante el periodo neonatal, la Pseudomona aeruginosa y el Staphylococus aureus pueden ser causa de OMA. Las enterobacterias aparecen en un $20 \%$ de las otitis del lactante de menos de 3 meses $^{4}$. En la mayor parte de los casos de OMA, el proceso infeccioso se resuelve con el tratamiento antibiótico. Sin embargo, la tomografía computarizada (TC) debe realizarse en forma temprana en el curso de la enfermedad para clasificar la mastoiditis como incipiente o coalescente. Además, la resonancia magnética (RM) es de utilidad cuando los hallazgos tomográficos son sugestivos de complicaciones intracraneales, debido a su alta sensibilidad para detectar colecciones extraxiales y problemas vasculares ${ }^{5}$.

El objetivo de esta presentación es resaltar el papel del diagnóstico temprano por imágenes, que permite a los médicos tratantes establecer 
una adecuada terapia y prevenir consecuencias de las complicaciones intracraneales.

\section{PRESENTACIÓN DEL CASO}

Paciente masculino, de 20 años de edad, que ingresa en el servicio de urgencias por traumatismo craneoencefálico moderado, como antecedentes de importancia tiene síndrome de Cornelia de Lange y fibrosis pulmonar.

Se le realiza una estudio de TC simple, donde se sugiere una colección parenquimatosa frontal derecha con extenso edema vasogénico frontoparietal, que produce un efecto de volumen sobre el ventrículo lateral y leve desviación de las estructuras de la línea media hacia la izquierda (Figs. 1A y B), además de la colección epidural hiperdensa por hematoma, localizado en la región temporal del mismo lado, con diámetros mayores de $18 \times 15 \times 5 \mathrm{~mm}$, con un leve efecto de volumen sobre el parénquima adyacente (Fig. 1 C). En la evaluación de la ventana ósea, no se visualizaron trazos de fractura. Se identifica ausencia de neumatización y esclerosis de las celdillas mastoideas derechas por cambios inflamatorios crónicos, con dos cavidades redondeadas en su espesor por coalescencia del proceso, que presentan dehiscencia del reborde posterior de la mastoides y que comunican con la cavidad craneal (flecha blanca) (Fig. 2A). Además se observa ocupación por material con densidad de tejido blando por tejido de granulación, que ocupa la totalidad de la cavidad del oído medio por cambios inflamatorios, con comunicación con las celdillas mastoideas a través del aditus ad antrum (flecha blanca) (Fig. 2B), asociado, se visualiza retracción de la membrana timpánica (flecha blanca) (Fig. 2C).
El paciente es valorado por el servicio de neurología donde se le prescribe tratamiento antibiótico con ceftriaxona y vancomicina por vía endovenosa, además de ampicilina por vía oral y glucocorticodes de administración nasal. Sin embargo, la evolución clínica del paciente empeora con fiebre y convulsiones tonicoclónicas, además presenta leucocitosis en el análisis de sangre, por lo que el servicio tratante inicia tratamiento anticonvulsivo por una semana y se decide realizar un estudio de RM cerebral contrastada, donde se evidencia una colección parenquimatosa frontal derecha, de paredes gruesas realzantes y septos en su interior, con diámetros mayores de $22 \times 20 \mathrm{~mm}$ en relación con el absceso, asociado con un extenso edema vasogénico y efecto de volumen sobre el parénquima cerebral adyacente y con una leve desviación de las estructuras de la línea media (Figs. 3 A-D). Además se identifica otra pequeña colección intraparenquimatosa de las mismas características, de localización temporal, del mismo lado, con diámetros mayores de $8 \times 7 \mathrm{~mm}$, con leve edema perilesional (Figs. 3 E y F). También se visualizan dos pequeñas colecciones tipo absceso en las celdillas mastoideas derechas (Fig. 4 A), además de defectos de llenado que comprometen el seno transverso, el sigmoide y la vena yugular interna derechos con realce de sus paredes, por el proceso de tromboflebitis (Figs. 4 B-F). Colección epidural de paredes gruesas realzantes con restricción a la difusión de su contenido por abscedación del hematoma, de localización temporal derecha con diámetros mayores de $39 \times 10$ mm (Figs. 5 A y B). También se identifica realce leptomeníngeo continuo anormal en el lóbulo temporal del mismo lado, por proceso de meningitis (Fig. 6). 

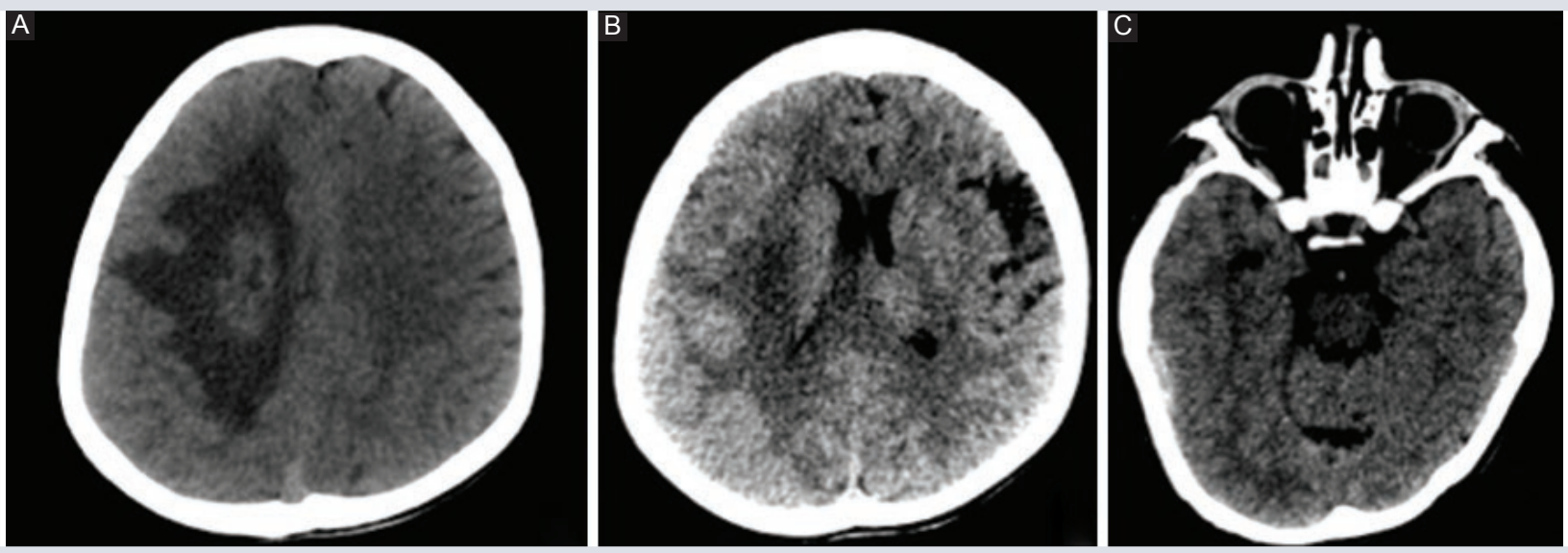

Figura 1. Tomografía de cráneo simple. A: cortes axiales a nivel de los centros semiovales, donde se sugiere presencia de una colección frontal derecha con un extenso edema vasogénico frontoparietal. B: cortes axiales a nivel de los cuerpos de los ventrículos laterales, donde se evidencia el efecto de volumen sobre el ventrículo lateral adyacente y una leve desviación de las estructuras de la línea media hacia la izquierda. C: cortes axiales a nivel de las órbitas, donde se visualiza una colección epidural hiperdensa en la región temporal derecha por hematoma, con un leve efecto de volumen sobre el parénquima cerebral adyacente.
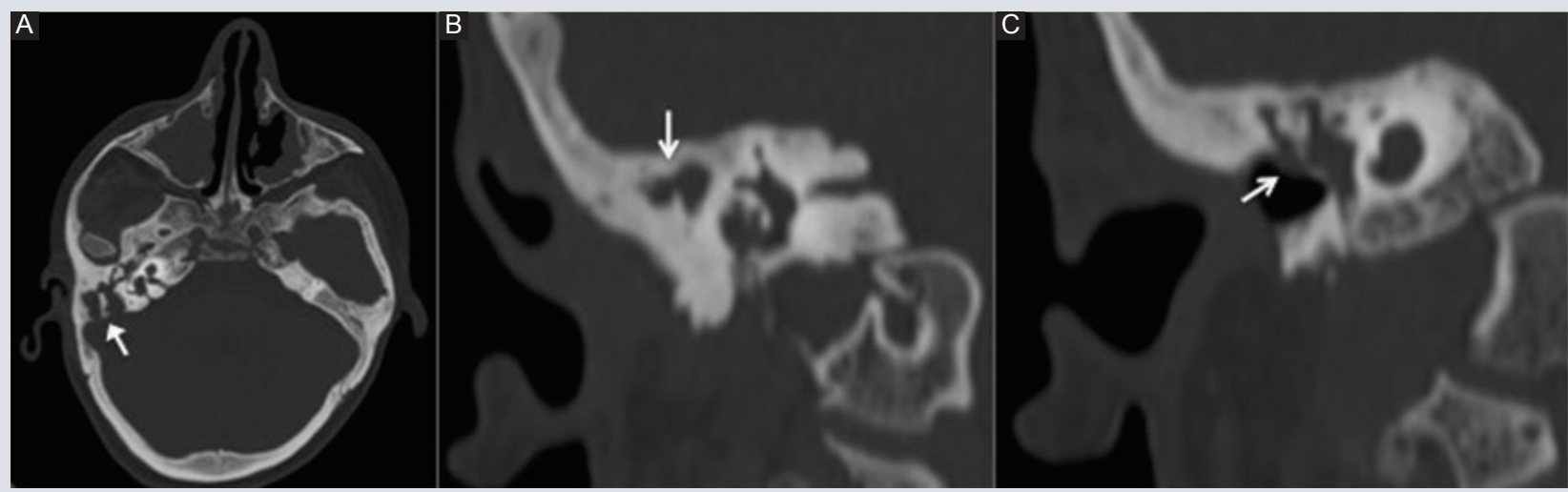

Figura 2. Tomografía de cráneo simple con ventana ósea. A: cortes axiales a nivel de los huesos temporales. Ausencia de neumatización y esclerosis de las celdillas mastoideas derechas por cambios inflamatorios crónicos, con dos cavidades redondeadas en su espesor por coalescencia del proceso, que presentan dehiscencia del reborde posterior de la mastoides y que comunican con la cavidad craneal (flecha). B: cortes coronales a nivel de los huesos temporales, donde se visualiza ocupación por material con densidad de tejido blando por tejido de granulación, que ocupa la totalidad de la cavidad del oído medio por cambios inflamatorios, comunicando con celdillas mastoideas a través del aditus ad antrum (flecha); C: cortes coronales a nivel de los huesos temporales, donde se se identifica retracción de la membrana timpánica asociada al proceso inflamatorio (flecha).

Se decide interconsultar al servicio de infectología, donde ordenan manejo antibiótico con meropenen y tratamiento anticoagulante, con lo que el paciente presenta mejoría clínica y de su estado neurológico, por lo que el servicio de neurocirugía decide no drenar quirúrgicamente las colecciones, además por el alto riesgo operatorio en relación con sus malformaciones relacionadas con su antecedente hereditario. 

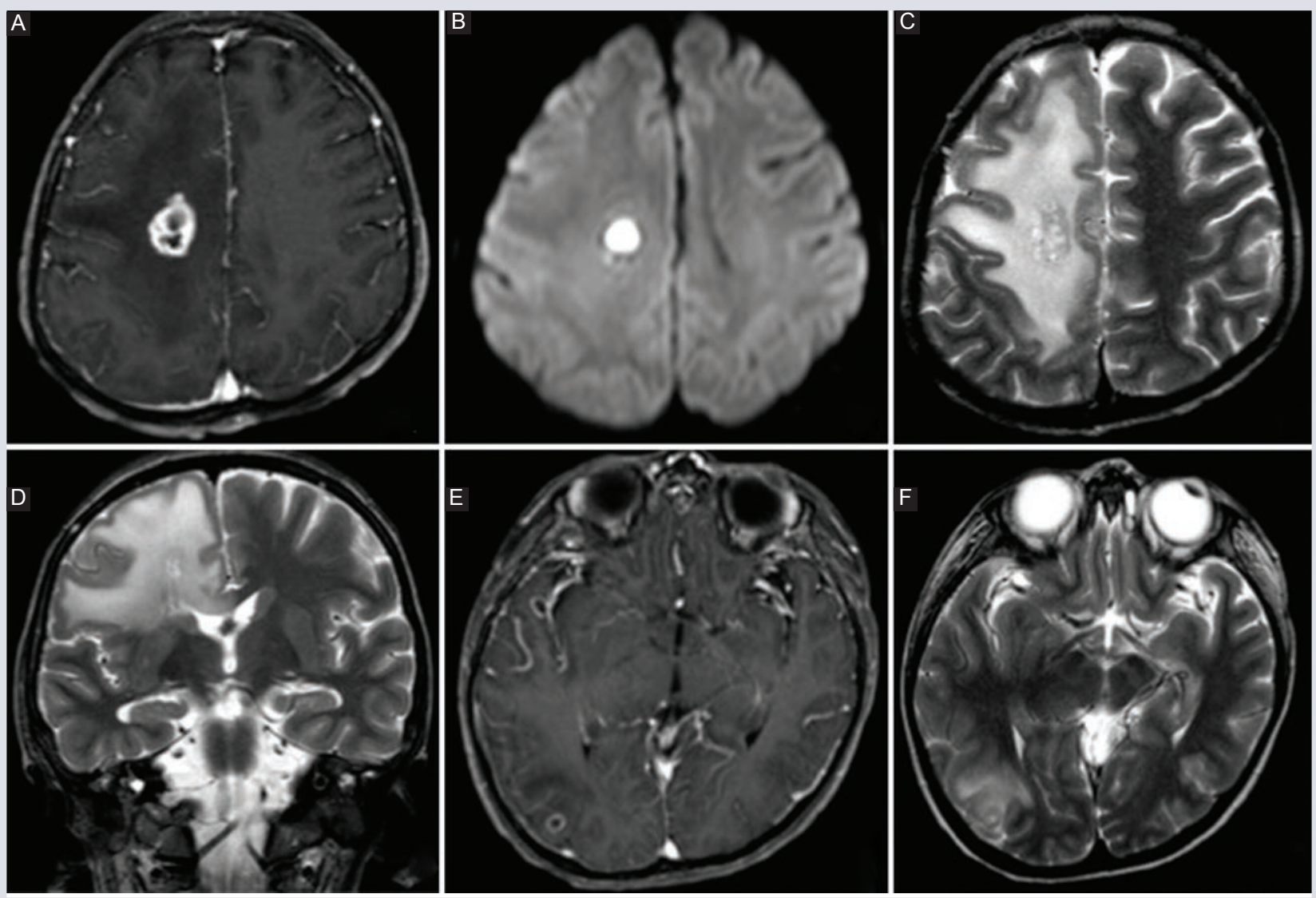

Figura 3. Resonancia magnética cerebral con contraste. A: imagen axial T1, donde se identifica colección parenquimatosa de paredes gruesas realzantes y septos en su interior en relación con el absceso frontal derecho. B: imagen axial sensible a la difusión, donde se demuestra que el contenido de la colección restringe a la difusión. C: imagen axial T2, donde se observa un extenso edema vasogénico perilesional. D: imagen coronal T2, donde se visualiza el efecto de volumen con leve desviación de estructuras de línea media hacia la izquierda. E: imagen axial T1, donde se identifica otra pequeña colección intraparenquimatosa redondeada con realce en anillo por absceso; F: localización temporal posterior derecha. E: leve edema perilesional.

\section{DISCUSIÓN}

La incidencia de complicaciones intracraneales de la otomastoiditis aguda ha aumentado recientemente debido a un tratamiento antibiótico insuficiente o inadecuado. Un alto índice de sospecha y un diagnóstico temprano son esenciales debido a la mortalidad asociada con estas complicaciones. Cuando se sospechan complicaciones intracraneales, el uso de TC de cráneo con medio de contraste intravenoso es crucial para su diagnóstico, sin embargo, la RM es de mayor sensibilidad y especificidad, ya que permite caracterizar mejor los procesos inflamatorios que parecen equívocos en la $\mathrm{TC}^{6}$.

El síndrome de Cornelia de Lange se caracteriza por rasgos faciales distintivos, retraso del crecimiento, hirsutismo y defectos por acortamiento en las extremidades superiores. La pérdida auditiva neurosensorial se observa en el $80 \%$ de los niños con síndrome de Cornelia de Lange, la cual se debe al mal funcionamiento de las estructuras del oído interno. 

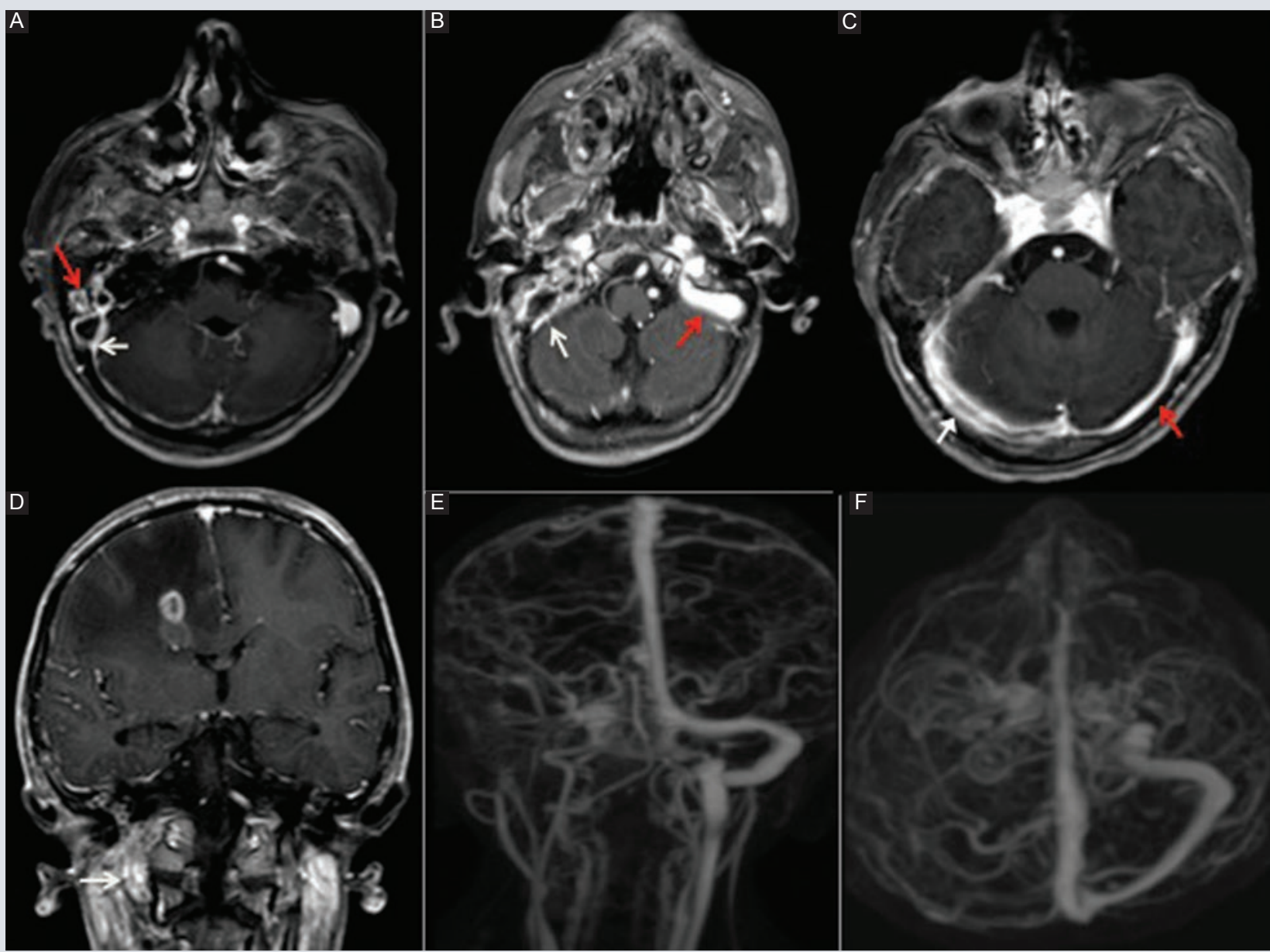

Fıgura 4. Resonancia magnética cerebral con contraste. A: imagen axial T1. Se visualizan dos pequeñas colecciones con realce en anillo tipo absceso en las celdillas mastoideas derechas (flecha roja), además de un defecto de llenado con realce de las paredes en la confluencia del seno transvero y sigmoide del mismo lado por tromboflebitis (flecha blanca). B: imagen axial T1. Se visualiza defecto de llenado con el contraste del seno sigmoide derecho con realce de las paredes (flecha blanca), el seno sigmoide contralateral presenta llenado satisfactorio (flecha roja). C: imagen axial T1, donde se observa defecto de llenado con el contraste del seno transverso derecho con realce de las paredes (flecha blanca), el seno transverso contralateral presenta llenado satisfactorio (flecha roja). D: imagen coronal T1. Se identifica defecto de llenado de la vena yugular interna derecha con realce de sus paredes (flecha blanca). E: imagen de venorrenosonancia con reconstrucción coronal MIP, donde se visualizan defectos de llenado en senos transverso y sigmoide, además en vena yugular interna del lado derecho. F: imagen de venorrenosonancia con reconstrucción axial MIP, donde se visualiza los defectos de llenado en los senos transverso y sigmoide, además de en la vena yugular interna del lado derecho. MIP: Proyección de Máxima Intensidad.

Además, la sepsis de origen neurológico se presenta en el $4 \%$ de los $\operatorname{casos}^{7}$.

Los abscesos cerebrales generalmente se consideran la segunda complicación intracraneal más frecuente de la otitis media después de la meningitis ${ }^{8}$. Históricamente, se ha informado que el 25\% de los abscesos cerebrales en niños es de origen otogénico, mientras que en los adultos se cree que más del 50\% de los abscesos cerebrales son de este origen ${ }^{9}$. Comienza con la colonización de microorganismos en el parénquima cerebral, que se visualizan en estudios de TC y RM como colecciones con cápsula vascularizada que presentan refuerzo de anillo bien definido ${ }^{10}$. Pueden ocurrir en la 

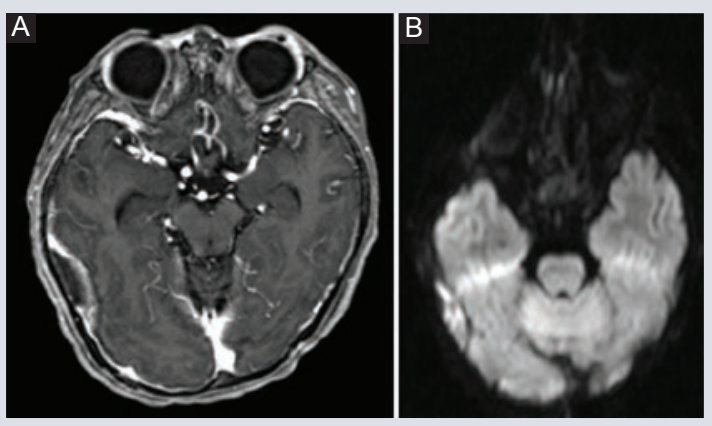

Figura 5. Resonancia magnética cerebral con contraste. A: imagen axial T1. Se evidencia colección epidural de las paredes gruesas realzantes de localización temporal derecha, que corresponde a un hematoma con abscedación secundaria y efecto de volumen sobre el parénquima cerebral adyacente.

B: imagen axial sensible a la difusión. Se identifica restricción a la difusión del contenido de la colección.

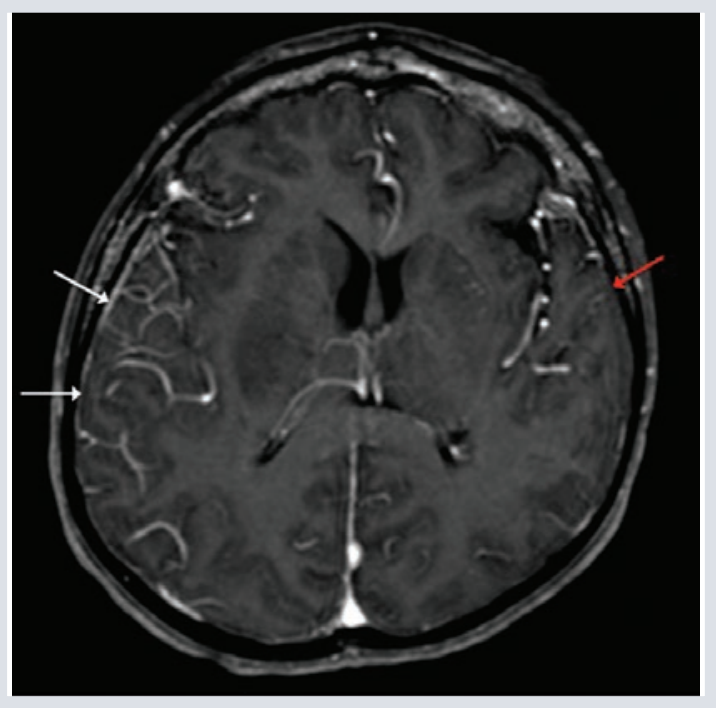

Figura 6. Resonancia magnética cerebral con contraste. Imagen axial T1, en donde se visualiza realce leptomeníngeo continuo anormal en la región temporal derecha (flechas blancas). Obsérvese el realce meníngeo normal en la región temporal contralateral (flecha roja).

fosa craneal media cuando hay destrucción del tegmen tympani y de la apófisis mastoides, o destrucción de la corteza anterior de la pirámide petrosa o de la corteza interna de los tractos de las células aéreas cigomáticas ${ }^{6}$.
El empiema subdural es una colección de material purulento entre la duramadre intracraneal y la aracnoides. El compartimento supratentorial es la ubicación más común, suelen verse en la convexidad y en la región interhemisférica. La tasa de mortalidad es de aproximadamente el 4\%. La morbilidad incluye hemiparesia en el 15 a 35\% y convulsiones persistentes en el 12 a $37.5 \%{ }^{8}$. Los empiemas epidurales son resultado de tromboflebitis y periflebitis. Generalmente se desarrollan en la fisura interhemisférica y a lo largo del tentorio. Se visualizan en imágenes de RM y TC como colecciones extraaxiales, con borde biconvexo (colección epidural) y con borde concavo (colección subdural), con realce periférico, además de efecto de volumen sobre el parénquima cerebral y los surcos adyacentes ${ }^{6}$. En la RM, estas colecciones presentan restricción de su contenido en la secuencia de difusión.

Los microorganismos pueden alcanzar las meninges a través de diferentes vías, como los tejidos blandos, áreas de erosión ósea, vías extradurales, laberinto auditivo o senos venosos infectados. En la TC o la RM se puede observar el realce meníngeo continuo anormal, indicativo de proceso de meningitis. Hay que tener en cuenta que la TC se debe realizar antes de la punción lumbar para el análisis del líquido cefalorraquídeo, para descartar signos de aumento de la presión intracraneal ${ }^{6}$.

La trombosis séptica del seno venoso transverso se observa como una complicación supurativa de la otitis media y la mastoiditis. Los gérmenes más frecuentes son el Proteus sp., E. coli y S. aureus. La mayoría de los pacientes se pueden presentar con parálisis facial y síntomas de hipertensión endocraneal. Además, 
en casos de trombosis de los senos sagitales, los pacientes pueden presentar alteraciones del estado mental, cambios en el sistema motor y parálisis facial ${ }^{10}$.

Otra complicación más rara es la trombosis séptica del seno cavernoso, que fue descrita por primera vez por Debase, en 1778. La fuente principal puede ser un foco distante con septicemia. Eloído medio suele ser de las causas menos frecuentes ${ }^{11}$.

Si bien la TC con contraste en fase venográfica no es el estudio de primera elección para el diagnóstico de esta entidad, es una opción para los pacientes con contraindicaciones para realizar un estudio de RM. En la TC cerebral simple, los hallazgos que pueden sugerir esta patología incluyen el «signo del triángulo», que consiste en una hiperdensidad triangular o redonda en la región posterior del seno sagital superior. El signo del «delta vacío» consiste en un defecto de llenado en el seno venoso comprometido, con patrón triangular de realce rodeándolo, en el estudio de TC con contraste endovenoso. Sin embargo, el estándar de oro para el diagnóstico hoy en día sigue siendo la RM contrastada ${ }^{1}$, en donde se evidencia fácilmente el defecto de llenado correspondiente al trombo en el seno venoso comprometido y con realce de sus paredes en el caso de tromboflebitis, además se puede demostrar con mayor detalle en las secuencias de venorresonancia y las correspondientes reconstrucciones tridimensionales.

\section{CONCLUSIÓN}

El caso clínico presentado en esta revisión es una muestra de la vigencia de las complicaciones intracraneales por patologías otogénicas, que históricamente han reducido de frecuencia, pero en las que persiste una elevada morbimortalidad.

La principal forma de controlar la otitis media y la mastoiditis crónica es el diagnóstico precoz, así como el tratamiento antibiótico y la intervención quirúrgica temprana en casos que se requiera, debido a que algunos patógenos microbianos facilitan la invasión y, consecuentemente, las complicaciones intracraneales mencionadas. Es importante conocer los patrones por TC y RM para un diagnóstico oportuno de estas complicaciones y establecer una planificación del tratamiento, con un abordaje multidisciplinario que incluya la interacción entre servicios de radiología, neurocirugía, neurología y microbiología.

Además, hay que tener en cuenta que ciertos acontecimientos, como síndromes genéticos o antecedentes de traumatismos, pueden disfrazar la clínica, lo que constituye un reto diagnóstico para el médico clínico tratante y dificulta el diagnóstico por imágenes.

\section{AGRADECIMIENTOS}

A los Médicos Radiólogos y Residentes, además del personal técnico y de enfermería de la División de Radiología e Imagen del Hospital Dr. Manuel Gea González.

\section{CONFLICTO DE INTERESES}

Los autores declaran que no hay conflictos de intereses con otras personas u organizaciones. 


\section{FINANCIAMIENTO}

Debido a que se trata de una presentación de caso no requirió de financiamiento.

\section{RESPONSABILIDADES ÉTICAS}

Protección de personas y animales. Los autores declaran que para esta investigación no se han realizado experimentos en seres humanos ni en animales.

Confidencialidad de los datos. Los autores declaran que han seguido los protocolos de su centro de trabajo sobre la publicación de datos de pacientes.

\section{Derecho a la privacidad y consentimiento} informado. Los autores han obtenido el consentimiento informado de los pacientes y/o sujetos referidos en el artículo. Este documento obra en poder del autor de correspondencia.

\section{BIBLIOGRAFÍA}

1. Kim SR, Choo OS, Park HY. Two cases of acute mastoiditis with subperiosteal abscess. Korean J Audiology. 2013;17(2):97.

2. Govea-Camacho LH, Pérez-Ramírez R, Cornejo-Suárez A, Fierro-Rizo R, Jiménez-Sala CJ, Rosales-Orozco CS. Diagnosis and treatment of the complications of otitis media in adults. Case series and literature review. Cir Cir. 2016;84(5):398-404.

3. López-Ladrón García de la Borbolla C, Ceballos Cauto M. Complicaciones de las otitis; En: Suárez C, Gil-carcedo LM, Marco J, Medina J, Ortega P, Trinidad J, eds. Libro virtual de formación en otorrinolaringología. 1. a ed. Madrid, España: SEORLPCF; 2014. p. 1-13.

4. James N, Marc DE. Infecciones del oído y de la mastoides. En: Hupp JR, Ferneini EM, ed. Infecciones orofaciales, de cabeza y cuello. 1. a ed. Elsevier; 2017. p. 293-303.

5. Elena P, Miguel M. Indications and radiological findings of acute otitis media and its complications. Sociedad Española de Otorrinolaringologia y Cirugía de Cabeza y Cuello. 2016;68(1):1-68.

6. Carcacia I, Prieto P, Quintero Rivera JC, Perez-Cid J, Corral Rivadulla RA, Carballo MC, et al. Acute mastoiditis and its complications. The role of imaging techniques: CT and MRI. ECR 2011;C-1063. p. 1-108.

7. Deardorff MA, Noon SE, MS, Krantz ID. Cornelia de Lange Syndrome. Gene Reviews. 2016;1-25.

8. Dattatraya M, Naresh B, Chandrashekhar D. Subdural empyema in children, Sistema Nervioso Infantil: ChNS. Diario Oficial de la Sociedad Internacional de Neurocirugía Pediátrica. 2018;34(10):1881-7.

9. Duarte MJ, Kozin ED, Barshak MB, Reinshagen K, Knoll RM, Abdullah KG, et al. Otogenic Brain Abscesses: A Systematic Review. Laryngoscope Investig Otolaryngol. 2018;3 (3):198-208.

10. Salih M, Ahmet C, Ozlem D, Gokhan B, Rahsan, Yesim C, et al, Mastoiditis, Brain Abscess and Sinus Thrombosis As Complications of Chronic Otitis Media: A Case Report. Jundishapur J Microbiol. 2017; 10(2):e41352.

11. Mahdieh A, Azadeh K, Mahbubeh T, Parisa A. Septic Cavernous Sinus Thrombosis: A Case Report. Iran Red Crescent Med J. 2016;18(8):e34961. 